\section{Acurácia da circunferência da cintura e da relação cintura/quadril como preditores de dislipidemias em estudo transversal de doadores de sangue de Cuiabá, Mato Grosso, Brasil}

\author{
Accuracy of waist circumference and \\ waist-to-hip ratio as predictors of dyslipidemia \\ in a cross-sectional study among blood donors \\ in Cuiabá, Mato Grosso State, Brazil
}

Márcia Gonçalves Ferreira 1 Joaquim Gonçalves Valente 2 Regina Maria Veras Gonçalves-Silva 1 Rosely Sichieri 2

\section{Introdução}

\author{
1 Departamento de \\ Alimentos e Nutrição, \\ Universidade Federal de \\ Mato Grosso, Cuiabá, Brasil. \\ 2 Departamento de \\ Epidemiologia, Universidade \\ do Estado do Rio de Janeiro, \\ Rio de Janeiro, Brasil. \\ Correspondência \\ M. G. Ferreira \\ Departamento de Alimento \\ e Nutrição, Universidade \\ Federal de Mato Grosso. \\ Av. Marechal Deodoro 829 \\ apto. 1202, Cuiabá, MT \\ 78005-505, Brasil. \\ margon@terra.com.br
}

\begin{abstract}
This study evaluated the accuracy of anthropometric fat location indices (waist circumference and waist-to-hip ratio) as predictors of dyslipidemia, in addition to defining their respective cut-offs. The sample consisted of 416 healthy male blood donors from 20 to 58 years of age at the Blood Center in Cuiabá, capital of Mato Grosso State, Brazil. Serum lipids were determined through the enzymatic method. Data analysis through the ROC curve identified the best indicator and the cut-off with the largest statistically significant area under the ROC curve. The best cut-off points for waist-to-hip ratio and waist circumference were 0.90 and $85 \mathrm{~cm}$, respectively. The area under the ROC curve between waist circumference and high cholesterol/HDL relation included the $0.50 \mathrm{val}$ ue, so waist circumference could not be considered a predictor of dyslipidemia. The best sensitivity and specificity were in the youngest group. Waist-to-hip ratio was a better predictor of dyslipidemia than waist circumference, although the latter showed better discriminating power to detect hypertriglyceridemia. The most accurate cut-off points were lower those set by the World Health Organization.
\end{abstract}

Anthropometry; Waist-hip Ratio; Dyslipidemia
Embora a Organização Mundial da Saúde (OMS) 1 preconize o uso da circunferência da cintura no ponto de corte de $94 \mathrm{~cm}$ para homens e $80 \mathrm{~cm}$ para mulheres, como medida de risco metabólico aumentado, poucos estudos no Brasil avaliaram a adequação do uso desse indicador, bem como os pontos de corte mais adequados para a população brasileira.

A obesidade e, particularmente, a localização abdominal de gordura tem grande impacto sobre as doenças cardiovasculares por associar-se com grande freqüência a condições tais como dislipidemias, hipertensão arterial, resistência à insulina e diabetes que favorecem a ocorrência de eventos cardiovasculares, particularmente os coronarianos 2,3. Independentemente do sobrepeso, a gordura abdominal é importante fator de risco para essas condições 4,5 .

Ainda que diversos métodos possam ser utilizados na caracterização da distribuição do tecido adiposo, os métodos mais acurados tais como a tomografia computadorizada e a ressonância magnética são de alto custo e de difícil execução, de forma que a antropometria tem sido muito aplicada para este fim, por meio da construção de índices antropométricos de boa acurácia, a partir de medidas antropométricas de fácil mensuração, obtidas a baixo custo.

A circunferência da cintura e a relação cintura/quadril são os indicadores mais utilizados 
na aferição da distribuição centralizada do tecido adiposo em avaliações individuais e coletivas 6 , contudo as diferenças na composição corporal dos diversos grupos etários e raciais dificultam o desenvolvimento de pontos de corte universais. O conhecimento desses pontos de corte é útil na detecção do risco de desenvolvimento de doenças, tanto na vigilância nutricional quanto em estudos de diagnóstico populacional. O objetivo deste estudo é identificar o melhor indicador antropométrico de localização de gordura e os melhores pontos de corte na predição do risco de dislipidemias, uma vez que estudo anterior dessa população 7 permitiu a identificação dos marcadores antropométricos de localização de gordura que mais fortemente associaram-se com as dislipidemias, levando-se em consideração potenciais fatores de confusão.

\section{Métodos}

\section{População de estudo}

Os dados apresentados referem-se aos doadores de sangue do Hemocentro Regional de Cuiabá, Mato Grosso, Brasil. Todos os doadores com idade de vinte anos ou mais, que se apresentaram neste hemocentro no período compreendido entre agosto de 1999 a janeiro de 2000 foram convidados a participar do estudo $(\mathrm{n}=$ 1.749). As mulheres foram excluídas por representarem uma parcela muito pequena dos doadores ( $n=249$ ), 12 indivíduos foram excluídos por fazerem uso regular de medicação, 15 por fazerem tratamento dietético, 15 alegaram perda de peso nos últimos dois meses anteriores à entrevista, 15 apresentaram Índice de Massa Corporal (IMC) menor do que 18,5kg/ $\mathrm{m}^{2}, 164$ tinham IMC maior do que $30 \mathrm{~kg} / \mathrm{m}^{2}, 29$ recusaram-se a participar da pesquisa e 15 ultrapassaram a capacidade de atendimento, restando 1.235 homens para o estudo, os quais foram entrevistados, sendo levantados dados demográficos, informações sobre o estilo de vida (tabagismo, atividade física, consumo de álcool) e dados relativos à composição corporal de todos eles. Um total de 416 homens retornaram em jejum de 8 a 12 horas para a realização dos exames bioquímicos. O protocolo de pesquisa foi aprovado pelo Comitê de Ética do Hospital Universitário Júlio Muller.

\section{Procedimentos de aferição}

\section{- Antropometria}

A medida da circunferência da cintura e do quadril foi realizada antes da doação de sangue, em duplicata, obedecendo à padronização de Callaway et al. 8. A aferição foi feita estando o indivíduo em pé, em posição ereta, utilizando-se uma fita métrica flexível e inextensível de $200 \mathrm{~cm}$ de comprimento, com precisão de uma casa decimal. Para garantir a validade e fidedignidade das medidas, observou-se rigorosamente a posição da fita no momento da medição, mantendo-a no plano horizontal. Para obtenção dos valores das circunferências, circundava-se com a fita o local do corpo que se desejava medir, sendo a mesma colocada com firmeza, sem esticar excessivamente, evitando-se assim a compressão do tecido subcutâneo. A leitura foi feita no centímetro mais próximo, no ponto de cruzamento da fita. As circunferências foram aferidas com o indivíduo usando apenas a roupa íntima, em posição ortostática, abdômen relaxado, braços ao lado do corpo e os pés juntos. A medida da circunferência da cintura foi tomada na altura da cintura natural do indivíduo, que é a parte mais estreita do tronco, e a circunferência do quadril foi medida na extensão máxima das nádegas.

\section{- Avaliação bioquímica e definição dos indicadores de dislipidemia}

A dosagem dos lipídios e lipoproteínas foi realizada no Laboratório de Patologia Clínica do Hospital Universitário Júlio Muller. O colesterol total, HDL-colesterol e os triglicérides foram determinados no soro, por método enzimático. De cada indivíduo foram retirados $5 \mathrm{ml}$ de sangue através de punção venosa. Após a coleta, o material foi deixado por aproximadamente $30 \mathrm{~min}$ em repouso e a seguir centrifugado por $10 \mathrm{~min}$ a $3.000 \mathrm{rpm}$. A determinação do colesterol total e triglicérides foi feita utilizando-se o equipamento AERONE 3200, e o HDL-colesterol foi determinado manualmente (Labtest Diagnóstica S/A, Belo Horizonte, Brasil).

\section{Análise dos dados}

Dos marcadores de dislipidemias, os mais associados a desenlaces desfavoráveis são os triglicérides elevados 9,10 e a relação colesterol/ HDL elevada 11,12. Estudo anterior, realizado nessa mesma população, também mostrou que a circunferência da cintura e a relação cintu- 
ra/quadril associaram-se positivamente aos triglicérides e à relação colesterol/HDL 7, sendo a dislipidemia definida como triglicérides $\geq 200 \mathrm{mg} / \mathrm{dl}$ e relação colesterol/HDL $\geq 5$ 13. No presente estudo, utilizamos esses mesmos indicadores de dislipidemia nos mesmos pontos de corte, conforme preconizado pela Sociedade Brasileira de Cardiologia (Valores de referência para diagnóstico das dislipidemias em adultos > 20 anos. http://www.consensos.hpg. ig.com.br, acessado em 26/Fev/2004).

Os pontos de corte para a circunferência da cintura e relação cintura/quadril foram determinados por meio de curvas ROC (Receiver Operating Characteristic), utilizando-se o pacote estatístico Stata/SE 7.0 (Stata Corporation, College Station, Estados Unidos). As curvas ROC são uma representação gráfica da sensibilidade (eixo vertical) e o complementar da especificidade (eixo horizontal) para diversos pontos de corte, que permitem identificar o melhor ponto de corte. Foram também determinados a área total sob a curva ROC e os intervalos de confiança (IC95\%). Na interpretação dos resultados considera-se que quanto maior a área sob a curva ROC, maior o poder de discriminação do indicador de obesidade central para dislipidemias, não devendo o limite inferior do intervalo de confiança atingir 0,50.

\section{Resultados}

Embora dois terços da população elegível não tenham voltado para realizar os exames bioquímicos, comparando-se os participantes com os não-participantes do estudo, não foram observadas diferenças estatisticamente significantes quanto às médias das variáveis antropométricas, com exceção do valor médio da relação cintura/quadril entre os mais jovens (idade $<31$ anos). Nesse grupo etário, a relação cintura/quadril mostrou-se maior para os que participaram do estudo em comparação aos que não participaram, ocorrendo o contrário com relação à variável renda (Tabela 1).

A prevalência de hipertrigliceridemia e da relação colesterol/HDL elevada foi menor nos pontos de corte para a circunferência da cintura e relação cintura/quadril propostos por este estudo, em comparação àqueles preconizados pela OMS 1 (Tabela 2). A relação cintura/quadril mostrou-se um melhor marcador de localização de gordura em comparação à circunferência da cintura na predição da relação colesterol/HDL elevada, considerada uma medida mais eficiente do que a hipertrigliceridemia na avaliação do risco cardiovascular. O melhor ponto de corte desse indicador para ambos os des-

Tabela 1

Média e erro padrão das variáveis antropométricas e sócio-demográficas segundo a idade.

\begin{tabular}{|c|c|c|c|c|c|}
\hline \multirow[t]{2}{*}{ Variável } & \multicolumn{2}{|c|}{ Participantes } & \multicolumn{2}{|c|}{ Não-participantes } & \multirow[t]{2}{*}{ p-valor } \\
\hline & Média & Erro padrão & Média & Erro padrão & \\
\hline \multicolumn{6}{|l|}{$<31$ anos } \\
\hline Índice de Massa Corporal (kg/m²) & 24,0 & 0,18 & 23,8 & 0,11 & 0,50 \\
\hline Gordura (\%) & 18,0 & 0,37 & 17,9 & 0,22 & 0,82 \\
\hline Circunferência da cintura $(\mathrm{cm})$ & 80,1 & 0,48 & 78,8 & 0,28 & 0,04 \\
\hline Relação cintura/quadril & 0,883 & 0,003 & 0,859 & 0,002 & $<0,001$ \\
\hline Renda $\left(\mathrm{smpc}^{\star \star}\right)$ & 2,0 & 0,12 & 2,8 & 0,14 & $<0,001$ \\
\hline Idade (anos) & 26,8 & 0,26 & 25,7 & 0,16 & 0,001 \\
\hline \multicolumn{6}{|l|}{$\geq 31$ anos } \\
\hline Índice de Massa Corporal (kg/m²) & 25,2 & 0,24 & 25,5 & 0,20 & 0,38 \\
\hline Gordura (\%) & 20,4 & 0,48 & 21,2 & 0,43 & 0,18 \\
\hline Circunferência da cintura $(\mathrm{cm})$ & 85,7 & 0,63 & 85,2 & 0,54 & 0,65 \\
\hline Relação cintura/quadril & 0,91 & 0,004 & 0,90 & 0,004 & 0,11 \\
\hline Renda $\left(\operatorname{smpc}^{\star \star}\right)$ & 1,9 & 0,19 & 3,5 & 0,43 & 0,02 \\
\hline Idade (anos) & 42,6 & 0,50 & 41,1 & 0,43 & 0,02 \\
\hline
\end{tabular}

* $\mathrm{p}$ valor associado ao teste de Mann Whitney;

** salário mínimo per capita. 
Prevalência de dislipidemias de acordo com a circunferência da cintura e relação cintura/quadril nos pontos de corte propostos pelo estudo ( $\geq 85$ e $\geq 0,90$, respectivamente) e nos pontos de corte preconizados pela OMS 1 ( $\geq 94$ e $\geq 1,0$, respectivamente).

\begin{tabular}{|c|c|c|c|c|c|c|c|c|c|c|c|c|}
\hline & \multicolumn{6}{|c|}{ Circunferência da cintura } & \multicolumn{6}{|c|}{ Relação cintura/quadril } \\
\hline & \multicolumn{3}{|c|}{$\geq 85$} & \multicolumn{3}{|c|}{$\geq 94$} & \multicolumn{3}{|c|}{$\geq 0,90$} & \multicolumn{3}{|c|}{$\geq 1,0$} \\
\hline & $\mathrm{n}$ & $\%$ & IC95\% & $\mathrm{n}$ & $\%$ & IC95\% & $n$ & $\%$ & IC95\% & $\mathrm{n}$ & $\%$ & IC95\% \\
\hline $\begin{array}{l}\text { Relação colesterol/ } \\
\text { HDL elevada }\end{array}$ & 20 & 13,2 & $8,2-19,6$ & 6 & 14,3 & $5,4-28,5$ & 23 & 13,8 & $8,9-19,9$ & 2 & 16,7 & $2,1-48,4$ \\
\hline Hipertrigliceridemia & 49 & 32,2 & $24,9-40,3$ & 16 & 38,1 & $23,6-54,4$ & 47 & 28,1 & $21,5-35,6$ & 4 & 33,3 & $9,9-65,1$ \\
\hline
\end{tabular}

fechos (hipertrigliceridemia e relação colesterol/HDL elevada) foi em torno de 0,90 (0,89 para relação colesterol/HDL elevada e 0,90 para hipertrigliceridemia). Na Figura 1 (a e b), estão representados esses pontos de corte e referidas as respectivas áreas sob a curva ROC para o total da população estudada ( 20 a 58 anos).

A circunferência da cintura associou-se apenas à hipertrigliceridemia, pois para a relação colesterol/HDL a área sob a curva incluiu o valor 0,50. A Figura 2 apresenta o melhor ponto de corte para a circunferência da cintura, referindose a área sob a curva ROC para hipertrigliceridemia, nos doadores de 20 a 58 anos de idade. Um total de $36,5 \%$ de indivíduos encontravam-se sob risco de desenvolvimento de hipertrigliceridemia pelo indicador circunferência da cintura e $40,0 \%$ apresentavam-se sob o risco de desenvolver hipertrigliceridemia e/ou relação colesterol/HDL elevada, quando se considera a relação cintura/quadril como marcador de risco. A sensibilidade e especificidade para cada indicador em relação aos desfechos são mostradas por faixa etária na Tabela 3. Os melhores valores de sensibilidade e especificidade foram encontrados no grupo etário mais jovem (20 a 30 anos).

\section{Discussão}

Diferentemente do proposto pela OMS 1, este estudo mostra que a circunferência da cintura não foi o melhor preditor de dislipidemias, embora tenha apresentado melhor acurácia que a relação cintura/quadril na detecção da hipertrigliceridemia. Além disso, os melhores pontos de corte definidos por meio da análise por curva ROC, tanto para a circunferência da cintura, quanto para a relação cintura/quadril mostraram-se inferiores aos que a OMS preconiza.

O padrão de distribuição da gordura e a composição corporal de forma geral variam grandemente de população para população 14,15. Nos últimos anos, um grande número de publicações tem evidenciado a inadequação de se aplicar pontos de corte definidos para populações caucasianas a outros grupos raciais. Um exemplo bastante nítido são os resultados que vêm sendo encontrados em populações asiáticas. Estudos que avaliaram os indicadores de localização de gordura em chineses e japoneses mostram claramente que os melhores pontos de corte para a detecção de diversas doenças crônicas estão abaixo daqueles recomendados pela OMS 16,17,18. Além disso, tem-se observado elevado porcentual de gordura corporal nesses indivíduos, apesar do IMC encontrarse dentro dos limites de normalidade 17.

No Brasil, poucas investigações avaliaram a associação entre indicadores antropométricos de localização de gordura e desfechos que se relacionam às doenças cardiovasculares 19,20,21, 22,23. Dentre esses estudos, apenas um 21 avaliou o melhor ponto de corte para a relação cintura/quadril em relação à hipertensão arterial, encontrando o valor 0,95 para homens, portanto, abaixo daquele preconizado pela OMS e próximo ao observado no nosso estudo, o que confirma a inadequação do uso de pontos de corte definidos para populações caucasianas quando se trata de avaliar a população brasileira.

A análise da sensibilidade e especificidade por meio da construção de curvas ROC tem sido recomendada em estudos epidemiológicos para a determinação de pontos de corte 24 . Esse tipo de análise permite não só a identificação do melhor ponto de corte como também fornece a área sob a curva que traduz o poder de discriminação de um indicador para um determinado desfecho. Nesse estudo, apenas a relação cintura/quadril apresentou capacidade preditiva para ambas as dislipidemias, ou seja, o IC95\% da área sob a curva não incluiu o valor 0,50 para nenhum dos desenlaces. No entanto, 
Figura 1

Relação cintura/quadril e dislipidemias (relação colesterol/HDL elevada e hipertrigliceridemia, respectivamente): sensibilidade, especificidade e melhor ponto de corte em doadores de sangue de 20 a 58 anos de idade, da Cidade de Cuiabá, Mato Grosso, Brasil.

Figura 1a

Relação colesterol/HDL elevada

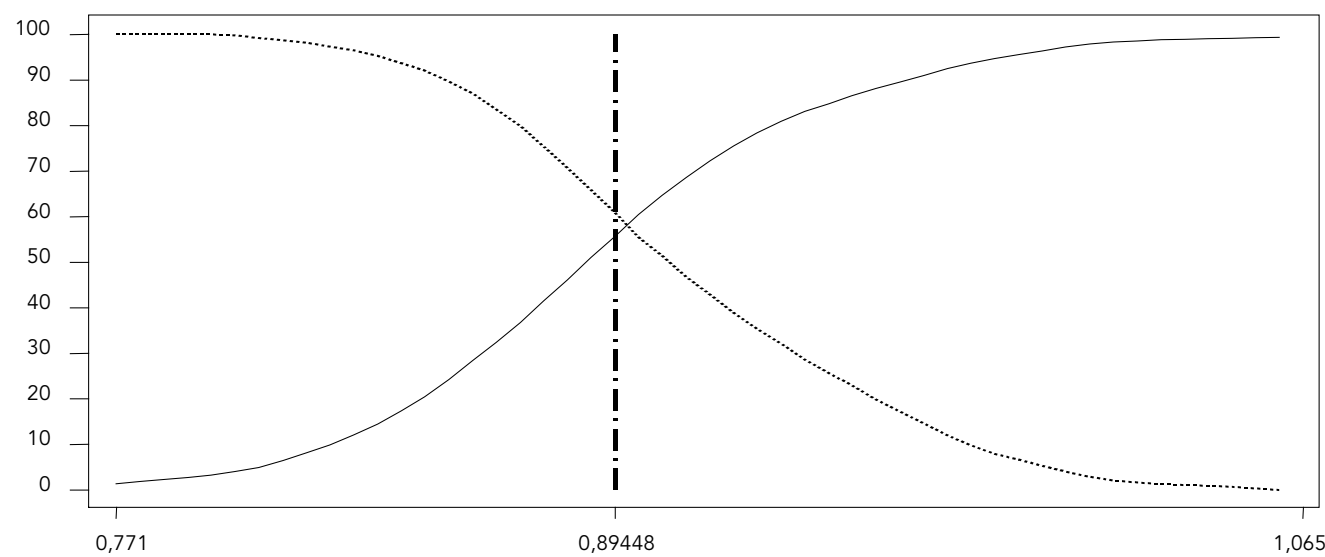

- especificidade …........ sensibilidade

Escore da variável rcq_bioq (teste de referência: colhdl_5) Curvas de sensibilidade e especificidade

Área sob a curva ROC: 0,6254 (IC95\%: 0,54158-0,70918).

Figura $1 b$

Hipertrigliceridemia

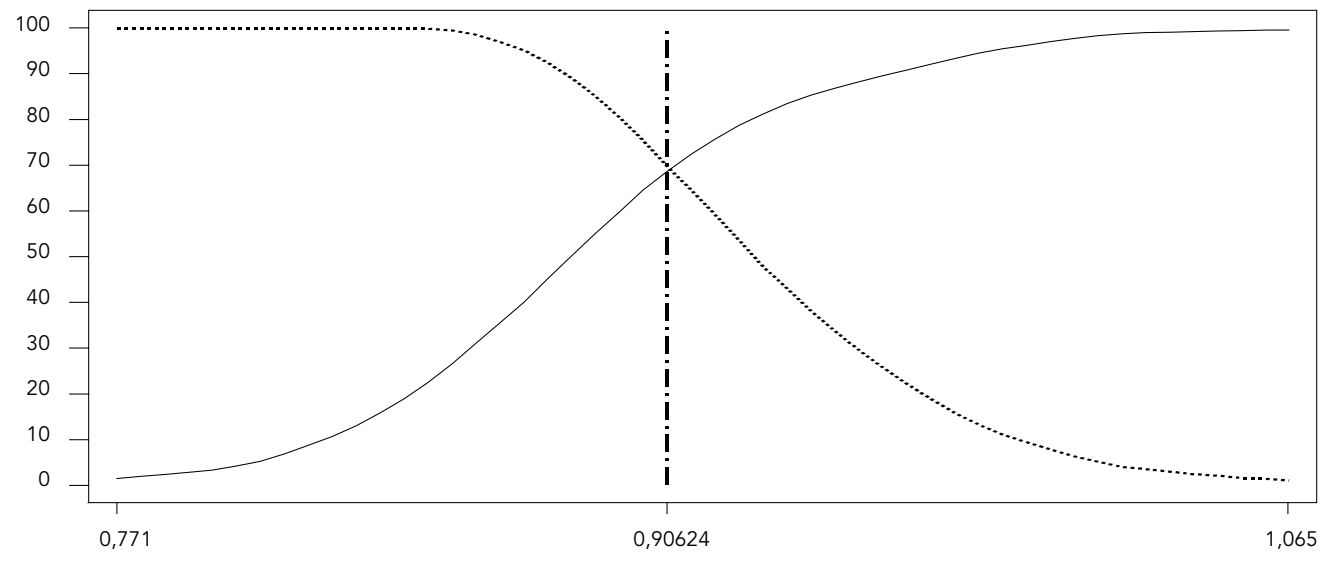

- especificidade

............ sensibilidade

Escore da variável rcq_bioq (teste de referência: triglic_200)

Curvas de sensibilidade e especificidade

Área sob a curva ROC: 0,7731 (IC95\%: 0,722242-0,82374). 
Figura 2

Circunferência da cintura e hipertrigliceridemia: sensibilidade, especificidade e melhor ponto de corte em doadores de sangue de 20 a 58 anos de idade, da Cidade de Cuiabá, Mato Grosso, Brasil.

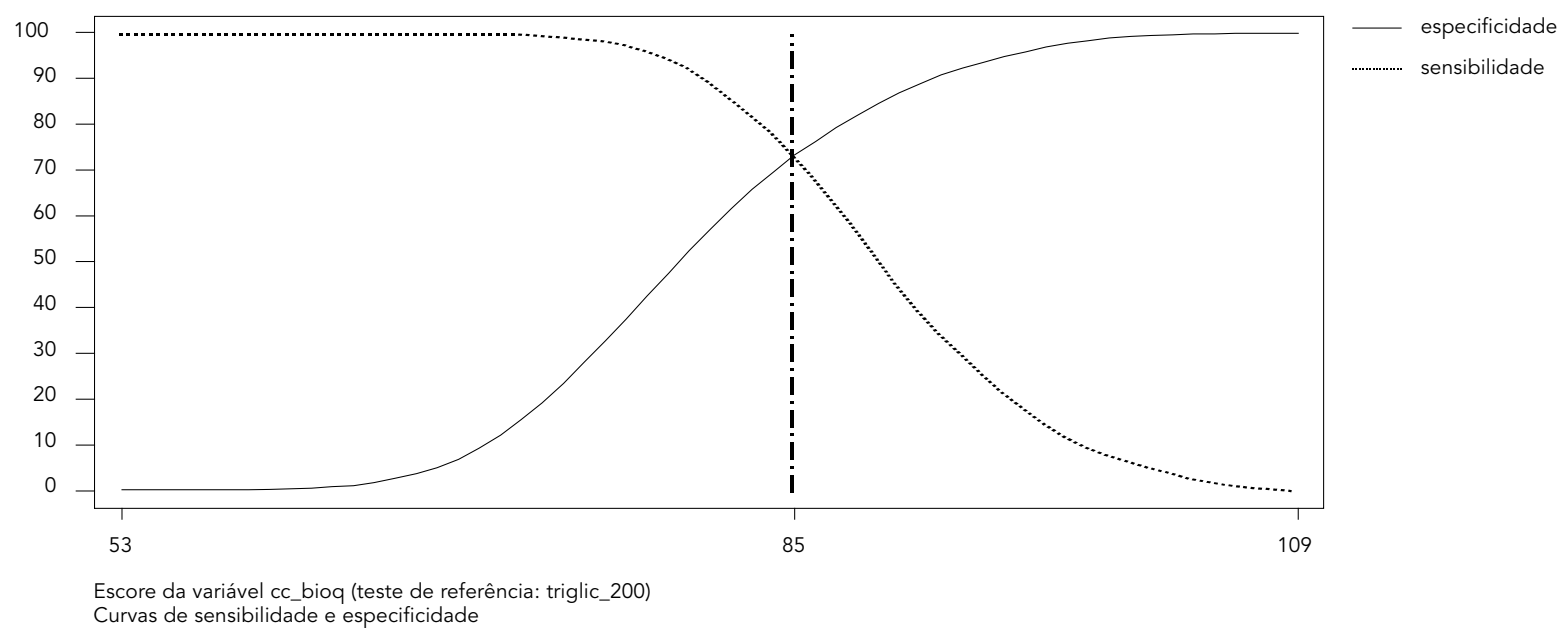

Área sob a curva ROC: 0,8268 (IC95\%: 0,78162-0,87194).

Tabela 3

Sensibilidade, especificidade e IC95\% dos indicadores circunferência da cintura e relação cintura/quadril

para os desfechos hipertrigliceridemia e relação colesterol/HDL elevada, segundo a idade.

\begin{tabular}{|c|c|c|c|c|c|c|c|c|}
\hline \multirow{2}{*}{ Indicadores } & \multicolumn{4}{|c|}{$20-30$ anos $(N=206)$} & \multicolumn{4}{|c|}{$31-58$ anos $(N=210)$} \\
\hline & $\begin{array}{c}\text { Sensibilidade } \\
(\%)\end{array}$ & IC95\% & $\begin{array}{c}\text { Especificidade } \\
(\%)\end{array}$ & IC95\% & $\begin{array}{c}\text { Sensibilidade } \\
\text { (\%) }\end{array}$ & IC95\% & $\begin{array}{c}\text { Especificidade } \\
(\%)\end{array}$ & IC95\% \\
\hline \multicolumn{9}{|c|}{ Circunferência da cintura } \\
\hline Hipertrigliceridemia & 69,2 & $38,6-90,9$ & 77,7 & $71,2-83,4$ & 68,1 & $52,9-80,9$ & 67,5 & $59,7-74,6$ \\
\hline \multicolumn{9}{|l|}{ Relação cintura/quadril } \\
\hline Hipertrigliceridemia & 84,6 & $54,6-98,1$ & 72,5 & $65,7-78,7$ & 66,0 & $50,7-79,1$ & 69,9 & $62,3-76,9$ \\
\hline $\begin{array}{l}\text { Relação colesterol/ } \\
\text { HDL elevada }\end{array}$ & 58,4 & $54,6-83,3$ & 59,5 & $52,2-66,4$ & 54,8 & $36,0-72,7$ & 56,0 & $48,3-63,3$ \\
\hline
\end{tabular}

nota-se que a circunferência da cintura mostrou melhor poder de detecção para a hipertrigliceridemia, com maior valor da área sob a curva ROC para esse desfecho $(0,82)$, quando comparada à relação cintura/quadril $(0,77)$.

Uma vantagem do presente estudo em relação a outros estudos transversais, foi a exclusão dos obesos da população de doadores de sangue, que representam um grupo populacional saudável ou sem doença reconhecida. A avaliação de marcadores antropométricos e seus pontos de corte em estudos transversais é sujeita a vieses, pelo conhecimento difundido de que a obesidade e as dislipidemias associam-se entre si e com as doenças cardiovasculares. Utilizar uma população de adultos saudáveis ou sem conhecimento de suas condições dislipidêmicas reduz a possibilidade de introdução desse viés.

Por outro lado, por tratar-se de uma população mais saudável, as prevalências de dislipidemias foram muito baixas, o que pode ter acarretado uma menor acurácia dos marcadores. Além disso, a taxa de não resposta para a reali- 
zação dos exames bioquímicos foi maior na renda mais baixa, onde as dislipidemias são mais freqüentes, o que pode ter causado viés no sentido oposto ao viés da população saudável. Contudo, a baixa prevalência de dislipidemia nas duas faixas de renda indica que o possível viés causado pela taxa de não resposta não seja importante. Um outro aspecto a ser considerado é que, por tratar-se de um estudo transversal, a análise de temporalidade fica comprometida, porém estudos longitudinais mostram associações similares àquelas observadas no presente estudo.

Considerando que o excesso de gordura na região central do corpo está associado ao aparecimento de doenças cardiovasculares 25,26,27,
28,29,30, diabetes 29,30,31, hospitalizações 32 e mortalidade 33,34 , a definição de pontos de corte para indicadores que se destacam por sua simplicidade operacional e boa acurácia permite a detecção dos indivíduos sob risco, sendo de grande utilidade nos serviços de atenção à saúde, além de possibilitar o conhecimento da situação de grupos populacionais específicos frente a esses riscos, quando empregados na pesquisa epidemiológica. Os resultados obtidos indicam a necessidade de serem realizados no Brasil estudos com diferentes desenlaces, em ambos os sexos, permitindo definir os melhores pontos de corte para os indicadores antropométricos de localização de gordura.

\section{Resumo}

Avaliou-se a acurácia dos indicadores antropométricos de localização de gordura (circunferência da cintura e relação cintura/quadril) como preditores de dislipidemias, apresentando seus pontos de corte. A amostra consistiu-se de 416 homens saudáveis, de 20 a 58 anos, doadores de sangue do hemocentro de Cuiabá, Mato Grosso, Brasil. Os lipídios séricos foram determinados por método enzimático. A análise dos dados foi feita através da curva ROC para identificar o melhor ponto de corte dos indicadores e a maior área estatisticamente significante sob a curva. Os melhores pontos de corte para relação cintura/quadril e circunferência da cintura foram 0,90 e $85 \mathrm{~cm}$, respectivamente. $O$ IC95\% da área sob a curva ROC entre a circunferência da cintura e a relação colesterol/HDL elevada incluiu o valor 0,50, limitando a capacidade da circunferência da cintura como preditor de dislipidemias. Valores de sensibilidade e especificidade foram melhores no grupo mais jovem. A relação cintura/quadril foi mais associada às dislipidemias do que a circunferência da cintura. Circunferência da cintura mostrou melhor poder discriminatório na detecção de hipertrigliceridemia. Os pontos de corte de melhor acurácia foram menores do que os estabelecidos pela Organização Mundial da Saúde.

Antropometria; Relação Cintura-quadril; Dislipidemia

\section{Colaboradores}

M. G. Ferreira participou como responsável pela coleta de dados em campo, executou as análises do artigo sob orientação de co-autores e redigiu o texto. J. G. Valente orientou o desenho do estudo e participou das análises do artigo. R. M. V. Gonçalves-Silva colaborou na coleta de dados e redação do artigo. R. Sichieri foi responsável pelo plano de análises e participou de todas as fases de produção do artigo.

\section{Referências}

1. World Health Organization. Obesity: preventing and managing the global epidemic. Geneva: World Health Organization; 1998. (WHO Technical Report Series 894).

2. Kannel WB, Wilson PW, Nam BH, D'Agostino RB. Risk stratification of obesity as a coronary risk factor. Am J Cardiol 2002; 90:697-701.

3. Tonstad S, Hjermann I. A high risk score for coronary heart disease is associated with the metabolic syndrome in 40-year-old men and women. J Cardiovasc Risk 2003; 10:129-35.

4. Bjorntorp P. Abdominal fat distribution and the metabolic syndrome. J Cardiovasc Pharmacol 1992; 20:526-8.

5. Sharma AM. Adipose tissue: a mediator of cardiovascular risk. Int J Obes Relat Metab Disord 2002; 26 Suppl 4:S5-7.

6. World Health Organization. Physical status: the use and interpretation of anthropometry. Geneva: World Health Organization; 1995. (WHO Technical Report Series 854).

7. Lemos-Santos MGF, Valente JG, Gonçalves-Silva, RMV, Sichieri R. Waist circumference and waistto-hip ratio as predictors of serum concentration of lipids in Brazilian men. Nutrition 2004; 20:85762.

8. Callaway CW, Chumlea WC, Bouchard C, Himes JH, Lohman TG, Martin AD, et al. Circumferences. In: Lohman TG, Roche AF, Martorell R, editors. Anthropometric standardization reference manual. Champaign: Human Kinetics Books; 1988. p. 39-54.

9. Iso H, Naito Y, Sato S, Kitamura A, Okamura T, Sankai T, et al. Serum triglycerides and risk of coronary heart disease among Japanese men and women. Am J Epidemiol 2001; 153:490-9.

10. de Sauvage Nolting PR, Defesche JC, Buirma RJ, Hutten BA, Lansberg PJ, Kastelein JJ. Prevalence and significance of cardiovascular risk factors in a large cohort of patients with familial hypercholesterolaemia. J Intern Med 2003; 253:161-8. 
11. Castelli WP. Cholesterol and lipids in the risk of coronary artery disease - the Framingham Heart Study. Can J Cardiol 1988; 4 (A Suppl):5A-10A.

12. Wilson PW, Anderson KM, Harris T, Kannel WB, Castelli WP. Determinants of change in total cholesterol and HDL-C with age: the Framingham Study. J Gerontol 1994; 49:M252-7.

13. Anonymous. National Cholesterol Education Program. Second Report of the Expert Panel on Detection, Evaluation, and Treatment of High Blood Cholesterol in Adults (Adult Treatment Panel II). Circulation 1994; 89:1333-445.

14. Lovejoy JC, Smith SR, Rood JC. Comparison of regional fat distribution and health risk factors in middle-aged white and African American women: the Healthy Transitions Study. Obes Res 2001; 9:10-6.

15. Okosun IS, Choi ST, Boltri JM, Parish DC, Chandra KM, Dever GE, et al. Trends of abdominal adiposity in white, black, and Mexican-American adults, 1988 to 2000. Obes Res 2003; 11:1010-7.

16. Lin WY, Lee LT, Chen CY, Lo H, Hsia HH, Liu IL, et al. Optimal cut-off values for obesity: using simple anthropometric indices to predict cardiovascular risk factors in Taiwan. Int J Obes Relat Metab Disord 2002; 26:1232-8.

17. Deurenberg-Yap M, Chew SK, Deurenberg P. Elevated body fat percentage and cardiovascular risks at low body mass index levels among Singaporean Chinese, Malays and Indians. Obes Rev 2002; 3:209-15.

18. Ito H, Nakasuga K, Ohshima A, Maruyama T, Kaji Y, Harada M, et al. Detection of cardiovascular risk factors by indices of obesity obtained from anthropometry and dual-energy X-ray absorptiometry in Japanese individuals. Int J Obes Relat Metab Disord 2003; 27:232-7.

19. Guedes DP, Guedes JERP. Distribuição de gordura corporal, pressão arterial e níveis de lipídioslipoproteínas plasmáticas. Arq Bras Cardiol 1998; 70:93-8.

20. Gus M, Moreira LB, Pimentel M, Gleisener ALM, Moraes RS, Fuchs FD. Associação entre diferentes indicadores de obesidade e prevalência de hipertensão arterial. Arq Bras Cardiol 1998; 70:111-4.

21. Pereira RA, Sichieri R, Marins VMR. Razão cintura/quadril como preditor de hipertensão arterial. Cad Saúde Pública 1999; 15:333-44.

22. Oliveira CL, Veiga GV, Sichieri R. Anthropometric markers for cardiovascular disease risk factors among overweight adolescents. Nutr Res 2001; 21:1335-45.

23. Olinto MTA, Nacul LC, Gigante DP, Costa JSD, Menezes AMB, Macedo S. Waist circumference as a determinant of hypertension and diabetes in Brazilian women: a population-based study. Public Health Nutr 2004; 7:629-35.
24. Erdreich LS, Lee ET. Use of relative operating characteristic analysis in epidemiology. A method for dealing with subjective judgement. Am J Epidemiol 1981; 114:649-62.

25. Grinker JA, Tucker KL, Vokonas PS, Rush D. Changes in patterns of fatness in adult men in relation to serum indices of cardiovascular risk: the Normative Aging Study. Int J Obes Relat Metab Disord 2000; 24:1369-78.

26. Dobbelsteyn CJ, Joffres MR, MacLean DR, Flowerdew G. A comparative evaluation of waist circumference, waist-to-hip ratio and body mass index as indicators of cardiovascular risk factors. The Canadian Heart Health Surveys. Int J Obes Relat Metab Disord 2001; 25:652-61.

27. Ho SC, Chen YM, Woo JLF, Leung SSF, Lam TH, Janus ED. Association between simple anthropometric indices and cardiovascular risk factors. Int J Obes Relat Metab Disord; 25:1689-97.

28. Doll S, Paccaud F, Bovet P, Burnier M, Wietlisbach V. Body mass index, abdominal adiposity and blood pressure: consistency of their association across developing and developed countries. Int J Obes Relat Metab Disord 2002; 26:48-57.

29. Huang KC, Lin WY, Lee LT, Chen CY, Lo H, Hsia $\mathrm{HH}$, et al. Four anthropometric indices and cardiovascular risk factors in Taiwan. Int J Obes Relat Metab Disord 2002; 26:1060-8.

30. Stolk RP, Meijer R, Mali WPTM, Grobbee DE, van der Graaf Y. Ultrasound measurements of intraabdominal fat estimate the metabolic syndrome better than do measurement of waist circumference. Am J Clin Nutr 2003; 77:857-60.

31. Lerario DDG, Gimeno SG, Franco LJ, Iunes M, Ferreira SRG, Grupo de Estudo de Diabetes na Comunidade Nipo-Brasileira. Excesso de peso e gordura abdominal para a síndrome metabólica em nipo-brasileiros. Rev Saúde Pública 2002; 36:4-11.

32. Afonso FM, Sichieri R. Associação do índice de massa corporal e da relação cintura/quadril com hospitalizações em adultos do Município do Rio de Janeiro, RJ. Rev Bras Epidemiol 2002; 5:153-63.

33. Kalmijn S, Curb JD, Rodriguez BL, Yano K, Abbott RD. The association of body weight and anthropometry with mortality in elderly men: the Honolulu Heart Program. Int J Obes Relat Metab Disord 1999; 23:395-402.

34. Bigaard J, Tjonneland A, Thomsen BL, Overvad K, Heitmann BL, Sorensen TI. Waist circumference, BMI, smoking, and mortality in middle-aged men and women. Obes Res 2003; 11:895-903.

Recebido em 03/Nov/2004 Versão final reapresentada em 01/Abr/2005 Aprovado em 30/Mai/2005 